\title{
Stable Equivalence over Symmetric Functions
}

\author{
William Y. C. Chen ${ }^{1}$ and Arthur L. B. Yang ${ }^{2}$ \\ Center for Combinatorics, LPMC \\ Nankai University, Tianjin 300071, P. R. China \\ Email: ${ }^{1}$ chen@nankai.edu.cn, ${ }^{2}$ yang@nankai.edu.cn \\ Submitted: Jun 30, 2005; Accepted: Nov 14, 2005; Published: Nov 22, 2005 \\ Mathematics Subject Classification: 05E05
}

Dedicated to Professor Richard P. Stanley on the Occasion of His Sixtieth Birthday

Abstract. By using cutting strips and transformations on outside decompositions of a skew diagram, we show that the Giambelli-type matrices for a given skew Schur function are stably equivalent to each other over symmetric functions. As a consequence, the Jacobi-Trudi matrix and the transpose of the dual Jacobi-Trudi matrix are stably equivalent over symmetric functions. This leads to an affirmative answer to a question proposed by Kuperberg.

Keywords: Giambelli-type matrix, Jacobi-Trudi matrix, dual Jacobi-Trudi matrix, stably equivalent, outside decomposition, cutting strip, twist transformation.

\section{Introduction}

In [3] Kuperberg introduced the notion of stable equivalence of matrices over a ring, under which the cokernel of a Kasteleyn or Kasteleyn-Percus matrix is invariant. Let $R$ be a commutative ring with unit. Let $M$ be an $n \times k$ matrix over $R$, and let $M^{T}$ denote the transpose of $M$. Recall that any matrix $M^{\prime}$ is called a stably equivalent form of $M$ if $M^{\prime}$ can be obtained from $M$ under the following operations: general row operations,

$$
M \longmapsto A M
$$

where $A$ is an $n \times n$ invertible matrix over $R$; general column operations,

$$
M \longmapsto M B
$$

where $B$ is a $k \times k$ invertible matrix over $R$; and stabilization

$$
M \longmapsto\left(\begin{array}{l|l}
1 & 0 \\
\hline 0 & \mathrm{M}
\end{array}\right)
$$

and its inverse.

This paper is motivated by Kuperberg's problem [3, Question 15] on the stable equivalence property between the Jacobi-Trudi matrix and the transpose of the dual Jacobi-Trudi 
matrix of skew Schur functions over the ring $\Lambda$ of symmetric functions. We assume that the reader is familiar with the notation and terminology of symmetric functions in [5]. Given a partition $\lambda$, let $\ell(\lambda)$ denote the length of $\lambda$. The Jacobi-Trudi matrix for the skew Schur function $s_{\lambda / \mu}$ is given by

$$
J_{\lambda / \mu}=\left(h_{\lambda_{i}-\mu_{j}-i+j}\right)_{i, j=1}^{\ell(\lambda)},
$$

where $h_{k}$ denotes the $k$-th complete symmetric function, $h_{0}=1$ and $h_{k}=0$ for $k<0$. The dual Jacobi-Trudi matrix for $s_{\lambda / \mu}$ is given by

$$
D_{\lambda / \mu}=\left(e_{\lambda_{i}^{\prime}-\mu_{j}^{\prime}-i+j}\right)_{i, j=1}^{\ell\left(\lambda^{\prime}\right)}
$$

where $\lambda^{\prime}$ is the partition conjugate to $\lambda, e_{k}$ denotes the $k$-th elementary symmetric function, $e_{0}=1$ and $e_{k}=0$ for $k<0$.

Theorem 14 of Kuperberg [3] states that the Jacobi-Trudi matrix and the dual JacobiTrudi matrix are stably equivalent over the polynomial ring. He asked whether they are stably equivalent over the ring of symmetric functions. But we note the the proof of [3, Thm. 14] actually shows that the Jacobi-Trudi matrix is stably equivalent to the transpose of the dual Jacobi-Trudi matrix. Consequently, Kuperberg's problem [3, Question 15] should be stated as follows:

Kuperberg's Question: Are $J_{\lambda / \mu}$ and $D_{\lambda / \mu}^{T}$ stably equivalent over the ring of symmetric functions?

In this paper, we will provide an affirmative answer to the above question. This paper is organized as follows. First we review some concepts of outside decompositions for a given skew diagram. Utilizing the cutting strips for a given edgewise connected skew shape as introduced by Chen, Yan and Yang [1], we demonstrate how a twist transformation changes the set of contents of the initial boxes of border strips in an outside decomposition, and how it changes the set of the contents of the terminal boxes. In Section 3, we construct the canonical form of the Giambelli-type matrix of the skew Schur function assuming that the outside decomposition is fixed. Using this canonical form we establish the stable equivalence property of the Giambelli-type matrix for the edgewise connected skew diagram. In Section 4, we show that for a general skew diagram the Jacobi-Trudi matrix and the transpose of its dual form are stably equivalent over the ring of symmetric functions.

\section{Twist transformations}

Let $\lambda$ be a partition of $n$ with $k$ parts, i.e., $\lambda=\left(\lambda_{1}, \lambda_{2}, \ldots, \lambda_{k}\right)$ where $\lambda_{1} \geq \lambda_{2} \geq \ldots \geq$ $\lambda_{k}>0$ and $\lambda_{1}+\lambda_{2}+\ldots+\lambda_{k}=n$. We represent $\lambda$ by its Young diagram: an array of boxes justified from the top and left corner with $k$ rows and $\lambda_{i}$ boxes in row $i$. A box $(i, j)$ in the diagram is the box in row $i$ from the top and column $j$ from the left. The content of $(i, j)$, denoted $\tau((i, j))$, is given by $j-i$. Given two partitions $\lambda$ and $\mu$, we say 
that $\mu \subseteq \lambda$ if $\mu_{i} \leq \lambda_{i}$ for all $i$. If $\mu \subseteq \lambda$, we define a skew partition $\lambda / \mu$, whose Young diagram is obtained from the Young diagram of $\lambda$ by peeling off the Young diagram of $\mu$ from the upper left corner. The conjugate of a skew partition $\lambda / \mu$, which we denote by $\lambda^{\prime} / \mu^{\prime}$, is defined to be the transpose of the skew diagram $\lambda / \mu$.

A skew diagram $\lambda / \mu$ is connected if it can be regarded as a union of an edgewise connected set of boxes, where two boxes are said to be edgewise connected if they share a common edge. A border strip is a connected skew diagram with no $2 \times 2$ block of boxes. If no two boxes lie in the same row, we call such a border strip a vertical border strip. If no two boxes lie in the same column, we call such a border strip a horizontal border strip. An outside decomposition of $\lambda / \mu$ is a partition of the boxes of $\lambda / \mu$ into pairwise disjoint border strips such that every border strip in the decomposition has a starting box on the left or bottom perimeter of the diagram and an ending box on the right or top perimeter of the diagram, see Figure 2.1 (d). This concept was used by Hamel and Goulden [2] to give a lattice path proof for the Giambelli-type determinant formulas for the skew Schur function.

Recall that a diagonal with content $c$ of $\lambda / \mu$ is the set of all the boxes in $\lambda / \mu$ having content $c$. Starting from the lower left corner of the skew diagram $\lambda / \mu$, we use consecutive integers $1,2, \ldots, d$ to number these diagonals. Chen, Yan and Yang [1] obtained the following characterization of outside decompositions of a skew shape.

Theorem 2.1 ([1, Theorem 2.2]) Suppose that $\lambda / \mu$ is an edgewise connected skew partition with d non-empty diagonals. Then there is a one-to-one correspondence between the outside decompositions of $\lambda / \mu$ and the set of border strips with $d$ boxes.

For each outside decomposition $\Pi$, the corresponding border strip $T$ is called the cutting strip of $\Pi$ in [1], which is given by the rule: for $i=1,2, \ldots, d-1$, the relative position between the $i$-th box and the $(i+1)$-st box in $T$ coincides with the relative position between the two boxes in the same border strip of $\Pi$, one of which is on the $i$-th diagonal and the other on the $(i+1)$-st diagonal, see Figure 2.1.

Notice that the relative position between the $i$-th box and the $(i+1)$-st box of the border strip imposes an up or right direction to the $i$-th box according to whether the $(i+1)$-st box lies above or to the right of the $i$-th box.

From the cutting strip characterization of outside decompositions, one can obtain any outside decomposition from another by a sequence of basic transformations of changing the directions of the boxes on a diagonal, which corresponds to the operation of changing the direction of a box in the cutting strip. This transformation is called the twist transformation on border strips.

Let $\lambda / \mu$ be an edgewise connected skew shape. Let $L$ be the diagonal of $\lambda / \mu$ consisting of the boxes with content $i$. Throughout this paper, we will read diagonals from the top left corner to the bottom right corner. Note that $L$ must be one of the four possible diagonal types classified by whether the first diagonal box has a box immediately above it, and whether the last diagonal box has a box immediately to its right. These four types are depicted by Figure 2.2. 


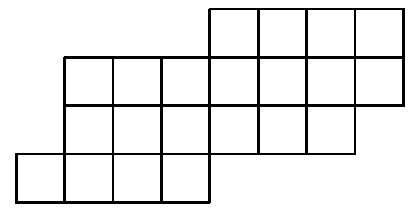

(a)

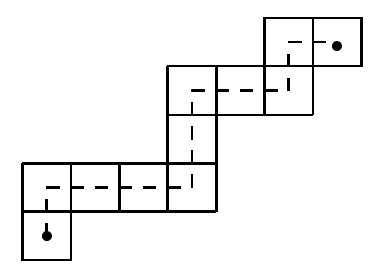

(c)

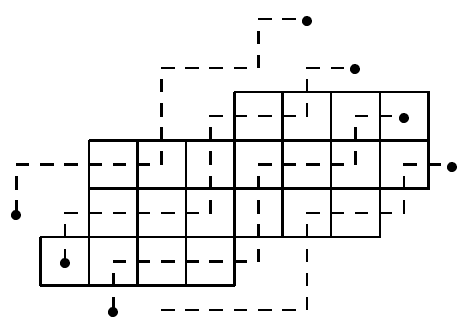

d $(\mathrm{b})$

$\Downarrow$

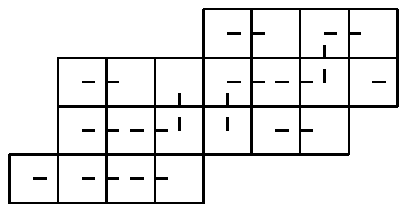

(d)

Figure 2.1 The cutting strip of an outside decomposition

Given an outside decomposition $\Pi=\left(\theta_{1}, \theta_{2}, \ldots, \theta_{m}\right)$ of $\lambda / \mu$ and a strip $\theta$ in $\Pi$, we denote the content of the initial box of $\theta$ and the content of the terminal box of $\theta$ respectively by $p(\theta)$ and $q(\theta)$. Let $\phi$ be the cutting strip of $\Pi$. It is known [1] that $\theta$ can be regarded as the segment of $\phi$ with the initial content $p(\theta)$ and the terminal content $q(\theta)$, denoted $\phi[p(\theta), q(\theta)]$.

Given two skew diagrams $I$ and $J$, let $I \triangleright J$ be the diagram obtained by gluing the lower left-hand corner box of diagram $J$ to the right of the upper right-hand corner box of diagram $I$, and let $I \uparrow J$ be the diagram obtained by gluing the lower left-hand corner box of diagram $J$ to the top of the upper right-hand corner box of diagram $I$. Suppose that the strip $\theta$ has a box in diagonal $L$. Then $\theta$ can be written as $\phi[p(\theta), i] \bullet \phi[i+1, q(\theta)]$ if $L$ has the right direction, and $\theta$ can be written as $\phi[p(\theta), i] \uparrow \phi[i+1, q(\theta)]$ if $L$ has the up direction.

Let $\omega_{i}$ denote the twist transformation acting on an outside decomposition $\Pi$ by changing the direction of the diagonal $L$. Let

$$
\begin{aligned}
P_{\Pi} & =\left\{p\left(\theta_{1}\right), p\left(\theta_{2}\right), \ldots, p\left(\theta_{m}\right)\right\}, \\
Q_{\Pi} & =\left\{q\left(\theta_{1}\right), q\left(\theta_{2}\right), \ldots, q\left(\theta_{m}\right)\right\} .
\end{aligned}
$$

The following theorem describes the actions of $\omega_{i}$ on $P_{\Pi}$ and $Q_{\Pi}$.

Theorem 2.2 Given an outside decomposition $\Pi$, let $\Pi^{\prime}$ be the outside decomposition obtained from $\Pi$ by applying the twist transformation $\omega_{i}$. Then we have

(a) $i \notin Q_{\Pi}, i+1 \notin P_{\Pi}, P_{\Pi^{\prime}}=P_{\Pi} \cup\{i+1\}$ and $Q_{\Pi^{\prime}}=Q_{\Pi} \cup\{i\}$, or

(b) $i \in Q_{\Pi}, i+1 \in P_{\Pi}, P_{\Pi^{\prime}}=P_{\Pi} \backslash\{i+1\}$ and $Q_{\Pi^{\prime}}=Q_{\Pi} \backslash\{i\}$, or 


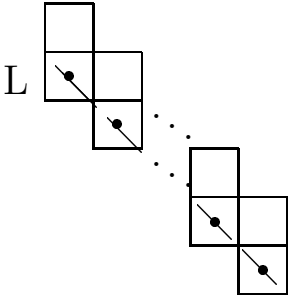

Type 1

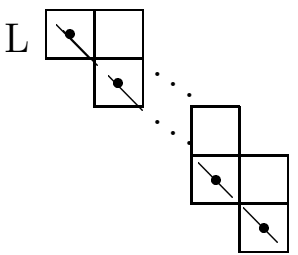

Type 3

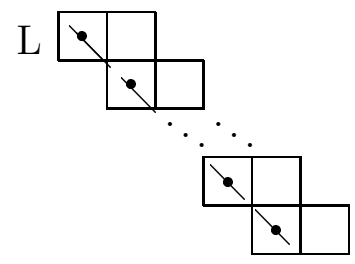

Type 2

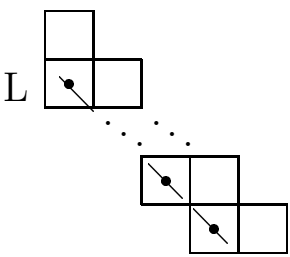

Type 4

Figure 2.2 Four possible types of diagonals of $\lambda / \mu$

(c) $i \in Q_{\Pi}, i+1 \notin P_{\Pi}, P_{\Pi^{\prime}}=P_{\Pi}$ and $Q_{\Pi^{\prime}}=Q_{\Pi}$, or

(d) $i \notin Q_{\Pi}, i+1 \in P_{\Pi}, P_{\Pi^{\prime}}=P_{\Pi}$ and $Q_{\Pi^{\prime}}=Q_{\Pi}$.

Proof. Suppose that $L$ has $r$ boxes. Since the twist transformation $\omega_{i}$ only changes the strips which contain a box in $L$, we may suppose that $\theta_{i_{t}}, 1 \leq t \leq r$, is the strip in $\Pi$ that contains the $t$-th diagonal box in $L$. Without loss of generality we may assume that the diagonal boxes have the up direction, since we can reverse the transformation process for the case when the diagonal boxes have the right direction.

Let $\phi^{\prime}$ be the cutting strip corresponding to the outside decomposition $\Pi^{\prime}$. Now we see the changes of $P_{\Pi}$ and $Q_{\Pi}$ under the action of the twist transformation $\omega_{i}$ according to the type of $L$ :

(a) If $L$ is of Type 1 , then we have $i \notin Q_{\Pi}$ and $i+1 \notin P_{\Pi}$. As illustrated in Figure 2.3, under the operation of $\omega_{i}$, the strip

$$
\theta_{i_{1}}=\phi\left[p\left(\theta_{i_{1}}\right), q\left(\theta_{i_{1}}\right)\right]=\phi\left[p\left(\theta_{i_{1}}\right), i\right] \uparrow \phi\left[i+1, q\left(\theta_{i_{1}}\right)\right]
$$

breaks into two strips

$$
\phi^{\prime}\left[p\left(\theta_{i_{1}}\right), q\left(\theta_{i_{2}}\right)\right]=\phi\left[p\left(\theta_{i_{1}}\right), i\right] \triangleright \phi\left[i+1, q\left(\theta_{i_{2}}\right)\right] \quad \text { and } \quad \phi^{\prime}\left[i+1, q\left(\theta_{i_{1}}\right)\right] .
$$

If $r>1$ then the last strip

$$
\theta_{i_{r}}=\phi\left[p\left(\theta_{i_{r}}\right), q\left(\theta_{i_{r}}\right)\right]=\phi\left[p\left(\theta_{i_{r}}\right), i\right] \uparrow \phi\left[i+1, q\left(\theta_{i_{r}}\right)\right]
$$


will be cut off into $\phi^{\prime}\left[p\left(\theta_{i_{r}}\right), i\right]$, and the other strips

$$
\theta_{i_{t}}=\phi\left[p\left(\theta_{i_{t}}\right), q\left(\theta_{i_{t}}\right)\right]=\phi\left[p\left(\theta_{i_{t}}\right), i\right] \uparrow \phi\left[i+1, q\left(\theta_{i_{t}}\right)\right], 2 \leq t \leq r-1
$$

will be twisted into

$$
\phi^{\prime}\left[p\left(\theta_{i_{t}}\right), q\left(\theta_{i_{t+1}}\right)\right]=\phi\left[p\left(\theta_{i_{t}}\right), i\right] \boldsymbol{\triangleright} \phi\left[i+1, q\left(\theta_{i_{t+1}}\right)\right] .
$$

Thus

$$
P_{\Pi^{\prime}}=P_{\Pi} \cup\{i+1\} \text { and } Q_{\Pi^{\prime}}=Q_{\Pi} \cup\{i\}
$$

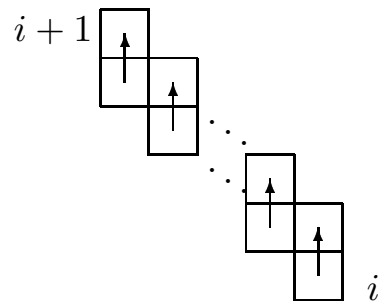

$L$

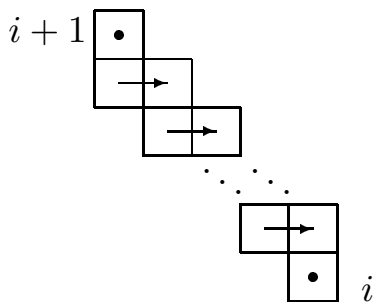

$\omega_{i}(L)$

Figure $2.3 \omega_{i}$ acts on a Type 1 diagonal $L$

(b) If $L$ is of Type 2, then we have $i \in Q_{\Pi}$ and $i+1 \in P_{\Pi}$. Let $\theta_{i_{r+1}}$ be the unique strip of $\Pi$ with the initial content $i+1$. Under the operation of $\omega_{i}$, the strip $\theta_{i_{1}}=\phi\left[p\left(\theta_{i_{1}}\right), i\right]$ becomes a part of the new strip

$$
\phi^{\prime}\left[p\left(\theta_{i_{1}}\right), q\left(\theta_{i_{2}}\right)\right]
$$

The strip $\theta_{i_{r+1}}=\phi\left[i+1, q\left(\theta_{i_{r+1}}\right)\right]$ becomes a part of the new strip

$$
\phi^{\prime}\left[p\left(\theta_{i_{r}}\right), q\left(\theta_{i_{r+1}}\right)\right]=\phi\left[p\left(\theta_{i_{r}}\right), i\right] \triangleright \phi\left[i+1, q\left(\theta_{i_{r+1}}\right)\right] .
$$

For $2 \leq t \leq r-1$, the strips

$$
\theta_{i_{t}}=\phi\left[p\left(\theta_{i_{t}}\right), q\left(\theta_{i_{t}}\right)\right]=\phi\left[p\left(\theta_{i_{t}}\right), i\right] \uparrow \phi\left[i+1, q\left(\theta_{i_{t}}\right)\right]
$$

will be twisted into

$$
\phi^{\prime}\left[p\left(\theta_{i_{t}}\right), q\left(\theta_{i_{t+1}}\right)\right]=\phi\left[p\left(\theta_{i_{t}}\right), i\right] \triangleright \phi\left[i+1, q\left(\theta_{i_{t+1}}\right)\right] .
$$

Thus

$$
P_{\Pi^{\prime}}=P_{\Pi} \backslash\{i+1\} \text { and } Q_{\Pi^{\prime}}=Q_{\Pi} \backslash\{i\} \text {. }
$$


(c) If $L$ is of Type 3, then we have $i \in Q_{\Pi}$ and $i+1 \notin P_{\Pi}$. Under the operation of $\omega_{i}$, the first strip $\theta_{i_{1}}=\phi\left[p\left(\theta_{i_{1}}\right), i\right]$ becomes

$$
\phi^{\prime}\left[p\left(\theta_{i_{1}}\right), q\left(\theta_{i_{2}}\right)\right]=\phi\left[p\left(\theta_{i_{1}}\right), i\right] \triangleright \phi\left[i+1, q\left(\theta_{i_{2}}\right)\right] .
$$

If $r>1$, the last strip

$$
\theta_{i_{r}}=\phi\left[p\left(\theta_{i_{r}}\right), q\left(\theta_{i_{r}}\right)\right]=\phi\left[p\left(\theta_{i_{r}}\right), i\right] \uparrow \phi\left[i+1, q\left(\theta_{i_{r}}\right)\right]
$$

will be cut off into $\phi^{\prime}\left[p\left(\theta_{i_{r}}\right), i\right]$, and the other strips

$$
\theta_{i_{t}}=\phi\left[p\left(\theta_{i_{t}}\right), q\left(\theta_{i_{t}}\right)\right]=\phi\left[p\left(\theta_{i_{t}}\right), i\right] \uparrow \phi\left[i+1, q\left(\theta_{i_{t}}\right)\right]
$$

will be twisted into

$$
\phi^{\prime}\left[p\left(\theta_{i_{t}}\right), q\left(\theta_{i_{t+1}}\right)\right]=\phi\left[p\left(\theta_{i_{t}}\right), i\right] \triangleright \phi\left[i+1, q\left(\theta_{i_{t+1}}\right)\right], 2 \leq t \leq r-1 .
$$

Thus

$$
P_{\Pi^{\prime}}=P_{\Pi} \text { and } Q_{\Pi^{\prime}}=Q_{\Pi}
$$

(d) If $L$ is of Type 4, then we have $i \notin Q_{\Pi}$ and $i+1 \in P_{\Pi}$. Let $\theta_{i_{r+1}}$ be the unique strip of $\Pi$ with the initial content $i+1$. Under the operation $\omega_{i}$, the first strip

$$
\theta_{i_{1}}=\phi\left[p\left(\theta_{i_{1}}\right), q\left(\theta_{i_{1}}\right)\right]=\phi\left[p\left(\theta_{i_{1}}\right), i\right] \uparrow \phi\left[i+1, q\left(\theta_{i_{1}}\right)\right]
$$

breaks into two strips

$$
\phi^{\prime}\left[p\left(\theta_{i_{1}}\right), q\left(\theta_{i_{2}}\right)\right]=\phi\left[p\left(\theta_{i_{1}}\right), i\right] \triangleright \phi\left[i+1, q\left(\theta_{i_{2}}\right)\right] \text { and } \phi^{\prime}\left[i+1, q\left(\theta_{i_{1}}\right)\right] \text {. }
$$

The strip $\theta_{i_{r+1}}$ becomes a part of the new strip

$$
\phi^{\prime}\left[p\left(\theta_{r}\right), q_{\theta_{r+1}}\right]=\phi\left[p\left(\theta_{r}\right), r\right] \triangleright \phi\left[i+1, q\left(\theta_{i_{r+1}}\right)\right] .
$$

The other strips

$$
\theta_{i_{t}}=\phi\left[p\left(\theta_{i_{t}}\right), q\left(\theta_{i_{t}}\right)\right]=\phi\left[p\left(\theta_{i_{t}}\right), i\right] \uparrow \phi\left[i+1, q\left(\theta_{i_{t}}\right)\right], 2 \leq t \leq r-1,
$$

will be twisted into

$$
\phi^{\prime}\left[p\left(\theta_{i_{t}}\right), q\left(\theta_{i_{t+1}}\right)\right]=\phi\left[p\left(\theta_{i_{t}}\right), i\right] \triangleright \phi\left[i+1, q\left(\theta_{i_{t+1}}\right)\right] .
$$

Thus

$$
P_{\Pi^{\prime}}=P_{\Pi} \text { and } Q_{\Pi^{\prime}}=Q_{\Pi}
$$




\section{Giambelli-type matrices for connected shapes}

By using the lattice path methodology, Hamel and Goulden [2] give a combinatorial proof for the Giambelli-type determinant formulas of the skew Schur function. In this section, we prove the stable equivalence of the Giambelli-type matrices of the Schur function indexed by an edgewise connected skew partition $\lambda / \mu$.

Given an outside decomposition $\Pi=\left(\theta_{1}, \theta_{2}, \ldots, \theta_{m}\right)$ of $\lambda / \mu$ and a strip $\theta$ in $\Pi$, let $\phi$ be the cutting strip of $\Pi$. Recall that the strip $\theta$ coincides with the segment $\phi[p(\theta), q(\theta)]$ of $\phi$. Following the treatment of [1], given any two contents $p, q$ we may define the strip $\phi[p, q]$ as follows:

(i) If $p \leq q$, then $\phi[p, q]$ is the segment of $\phi$ starting with the box having content $p$ and ending with the box having content $q$;

(ii) If $p=q+1$, then $\phi[p, q]$ is the empty strip $\emptyset$.

(iii) If $p>q+1$, then $\phi[p, q]$ is undefined.

Hamel and Goulden proved the following result.

Theorem 3.1 ([2, Theorem 3.1]) The skew Schur function $s_{\lambda / \mu}$ can be evaluated by the following determinant:

$$
D(\Pi)=\operatorname{det}\left(s_{\phi\left[p\left(\theta_{i}\right), q\left(\theta_{j}\right)\right]}\right)_{i, j=1}^{m}
$$

where $s_{\emptyset}=1$ and $s_{\text {undefined }}=0$.

Let us denote the Giambelli-type matrix in (3.5) by $M(\Pi)$. Chen, Yan and Yang [1] have obtained the canonical form of $M(\Pi)$ :

$$
C(\Pi)=\left(s_{\phi\left[p_{i}, q_{j}\right]}\right)_{i, j=1}^{m},
$$

where the sequence $\left(p_{1}, p_{2}, \ldots, p_{m}\right)$ is the decreasing reordering of $\left(p\left(\theta_{1}\right), p\left(\theta_{2}\right), \ldots\right.$, $\left.p\left(\theta_{m}\right)\right)$ and $\left(q_{1}, q_{2}, \ldots, q_{m}\right)$ is the decreasing reordering of $\left(q\left(\theta_{1}\right), q\left(\theta_{2}\right), \ldots, q\left(\theta_{m}\right)\right)$. It is clear that if $s_{\left[p_{i}, q_{j}\right]}=0$ then $s_{\left[p_{i}, q_{j^{\prime}}\right]}=0$ and $s_{\left[p_{i^{\prime}}, q_{j}\right]}=0$ for $j \leq j^{\prime} \leq m$ and $1 \leq i^{\prime} \leq i$.

Since $M(\Pi)$ and $C(\Pi)$ can be obtained from each other by permutations of rows and columns, we have

Lemma 3.2 For an outside decomposition $\Pi$ of the skew diagram $\lambda / \mu$, the two matrices $M(\Pi)$ and $C(\Pi)$ are stably equivalent over the ring $\Lambda$ of symmetric functions.

In order to show that the two Giambelli-type matrices $M(\Pi)$ and $M\left(\Pi^{\prime}\right)$ are stably equivalent over $\Lambda$, it suffices to prove that their canonical forms $C(\Pi)$ and $C\left(\Pi^{\prime}\right)$ are stably equivalent. To this end, we need the following lemma, which follows from the combinatorial definition of Schur functions and is proved, for example, in $[4,6]$ : 
Lemma 3.3 Let I and $J$ be two skew diagrams. Then

$$
s_{I} s_{J}=s_{I \triangleright J}+s_{I \uparrow J} .
$$

We now come to the main theorem of this paper:

Theorem 3.4 Let $\Pi$ and $\Pi^{\prime}$ be two outside decompositions of the edgewise connected skew diagram $\lambda / \mu$. Then the Giambelli-type matrices $M(\Pi)$ and $M\left(\Pi^{\prime}\right)$ are stably equivalent over the ring $\Lambda$ of symmetric functions.

Proof. By Lemma 3.2, we only need to prove that $C(\Pi)$ and $C\left(\Pi^{\prime}\right)$ are stably equivalent over $\Lambda$. Since any two outside decompositions can be obtained from each other by a sequence of twist transformations, it suffices to prove the case when $\Pi^{\prime}=\omega_{i}(\Pi)$ for any twist transformation $\omega_{i}$. Let $\phi$ be the cutting strip of $\Pi$, and let $\phi^{\prime}$ be the cutting strip of $\Pi^{\prime}$. We will only give the arguments for the case that the box of content $i$ in $\phi$ has the up direction. The case that the box of content $i$ in the cutting strip $\phi$ has the right direction can be dealt as the case that the box of content $i$ in the cutting strip $\phi^{\prime}$ has the up direction. As in Theorem 2.2, there are four cases:

(a) $i \notin Q_{\Pi}, i+1 \notin P_{\Pi}, P_{\Pi^{\prime}}=P_{\Pi} \cup\{i+1\}$ and $Q_{\Pi^{\prime}}=Q_{\Pi} \cup\{i\}$. Suppose that $k$ and $k^{\prime}$ are the two indices such that

$$
p_{k}>i+1 \text { and } p_{k+1}<i+1 \text {; while } q_{k^{\prime}}>i \text { and } q_{k^{\prime}+1}<i \text {. }
$$

Then the canonical matrix $C(\Pi)$ has the following form

$$
\left(\begin{array}{cccccc}
s_{\phi\left[p_{1}, q_{1}\right]} & \cdots & s_{\phi\left[p_{1}, q_{k^{\prime}}\right]} & 0 & \cdots & 0 \\
\vdots & \vdots & \vdots & \vdots & \vdots & \vdots \\
s_{\phi\left[p_{k}, q_{1}\right]} & \cdots & s_{\phi\left[p_{k}, q_{k^{\prime}}\right]} & 0 & \cdots & 0 \\
s_{\phi\left[p_{k+1}, i\right] \uparrow \phi\left[i+1, q_{1}\right]} & \cdots & s_{\phi\left[p_{k+1}, i\right] \uparrow \phi\left[i+1, q_{k^{\prime}}\right]} & \left.s_{\phi\left[p_{k+1}, q_{k^{\prime}+1}\right]}\right] & \cdots & s_{\phi\left[p_{k+1}, q_{m}\right]} \\
\vdots & \vdots & \vdots & \vdots & \vdots & \vdots \\
s_{\phi\left[p_{m}, i\right] \uparrow \phi\left[i+1, q_{1}\right]} & \cdots & s_{\phi\left[p_{m}, i\right] \uparrow \phi\left[i+1, q_{k^{\prime}}\right]} & s_{\phi\left[p_{m}, q_{k^{\prime}+1}\right]} & \cdots & s_{\phi\left[p_{m}, q_{m}\right]}
\end{array}\right),
$$

and the canonical matrix $C\left(\Pi^{\prime}\right)$ has the following form

$$
\left(\begin{array}{ccccccc}
s_{\phi\left[p_{1}, q_{1}\right]} & \cdots & s_{\phi\left[p_{1}, q_{k^{\prime}}\right]} & 0 & 0 & \cdots & 0 \\
\vdots & \vdots & \vdots & \vdots & \vdots & \vdots & \vdots \\
s_{\phi\left[p_{k}, q_{1}\right]} & \cdots & s_{\phi\left[p_{k}, q_{k^{\prime}}\right]} & 0 & 0 & \cdots & 0 \\
s_{\phi\left[i+1, q_{1}\right]} & \cdots & s_{\phi\left[i+1, q_{k^{\prime}}\right]} & 1 & 0 & \cdots & 0 \\
s_{\phi\left[p_{k+1}, i\right] \triangleright \phi\left[i+1, q_{1}\right]} & \cdots & s_{\phi\left[p_{k+1}, i\right] \triangleright \phi\left[i+1, q_{k^{\prime}}\right]} & s_{\phi\left[p_{k+1}, i\right]} & s_{\phi\left[p_{k+1}, q_{k^{\prime}+1}\right]} & \cdots & s_{\phi\left[p_{k+1}, q_{m}\right]} \\
\vdots & \vdots & \vdots & \vdots & \vdots & \vdots & \vdots \\
s_{\phi\left[p_{m}, i\right] \triangleright \phi\left[i+1, q_{1}\right]} & \cdots & s_{\phi\left[p_{m}, i\right] \triangleright \phi\left[i+1, q_{k^{\prime}}\right]} & s_{\phi\left[p_{m}, i\right]} & s_{\phi\left[p_{m}, q_{k^{\prime}+1}\right]} & \cdots & s_{\phi\left[p_{m}, q_{m}\right]}
\end{array}\right) .
$$

For $j: 1 \leq j \leq k^{\prime}$ subtracting the $\left(k^{\prime}+1\right)$-st column of $C\left(\Pi^{\prime}\right)$ multiplied by $s_{\phi\left[i+1, q_{j}\right]}$ from the $j$-th column, then for $j: k+2 \leq j \leq m+1$, subtracting the $(k+1)$-st row 
multiplied by $s_{\phi\left[p_{j-1}, i\right]}$ from the $j$-th row, we get the following matrix due to Lemma 3.3

$$
\left(\begin{array}{ccccccc}
s_{\phi\left[p_{1}, q_{1}\right]} & \cdots & s_{\phi\left[p_{1}, q_{k^{\prime}}\right]} & 0 & 0 & \cdots & 0 \\
\vdots & \vdots & \vdots & \vdots & \vdots & \vdots & \vdots \\
s_{\phi\left[p_{k}, q_{1}\right]} & \cdots & s_{\phi\left[p_{k}, q_{k^{\prime}}\right]} & 0 & 0 & \cdots & 0 \\
0 & \cdots & 0 & 1 & 0 & \cdots & 0 \\
-s_{\phi\left[p_{k+1}, i\right] \uparrow \phi\left[i+1, q_{1}\right]} & \cdots & -s_{\phi\left[p_{k+1}, i\right] \uparrow \phi\left[i+1, q_{k^{\prime}}\right]} & 0 & s_{\phi\left[p_{k+1}, q_{k^{\prime}+1}\right]} & \cdots & s_{\phi\left[p_{k+1}, q_{m}\right]} \\
\vdots & \vdots & \vdots & \vdots & \vdots & \vdots & \vdots \\
-s_{\phi\left[p_{m}, i\right] \uparrow \phi\left[i+1, q_{1}\right]} & \cdots & -s_{\phi\left[p_{m}, i\right] \uparrow \phi\left[i+1, q_{k^{\prime}}\right]} & 0 & s_{\phi\left[p_{m}, q_{k^{\prime}+1}\right]} & \cdots & s_{\phi\left[p_{m}, q_{m}\right]}
\end{array}\right) .
$$

By multiplying -1 for the last $m-k$ rows and the last $m-k^{\prime}$ columns, then permuting rows and columns, and the inverse operation of stabilization, we find that the above matrix is stably equivalent to $C(\Pi)$ over the ring $\Lambda$ of symmetric functions. Thus $C(\Pi)$ and $C\left(\Pi^{\prime}\right)$ are stably equivalent over $\Lambda$.

(b) $i \in Q_{\Pi}, i+1 \in P_{\Pi}, P_{\Pi^{\prime}}=P_{\Pi} \backslash\{i+1\}$ and $Q_{\Pi^{\prime}}=Q_{\Pi} \backslash\{i\}$. In this case, we only need to reverse the process of the operations of case $(\mathrm{a})$, where $\omega_{i}$ is now regarded as a transformation from the right direction to the up direction. Notice that each inverse operation is still over the ring $\Lambda$ of symmetric functions. Thus $C(\Pi)$ and $C\left(\Pi^{\prime}\right)$ are stably equivalent over $\Lambda$.

(c) $i \in Q_{\Pi}, i+1 \notin P_{\Pi}, P_{\Pi^{\prime}}=P_{\Pi}$ and $Q_{\Pi^{\prime}}=Q_{\Pi}$. Suppose that $k$ and $k^{\prime}$ are the two indices such that

$$
p_{k}>i+1 \text { and } p_{k+1}<i+1 ; \text { while } q_{k^{\prime}}=i \text {. }
$$

Then the canonical matrix $C(\Pi)$ has the following form

$$
\left(\begin{array}{ccccccc}
s_{\phi\left[p_{1}, q_{1}\right]} & \cdots & s_{\phi\left[p_{1}, q_{k^{\prime}-1}\right]} & 0 & 0 & \cdots & 0 \\
\vdots & \vdots & \vdots & \vdots & \vdots & \vdots & \vdots \\
s_{\phi\left[p_{k}, q_{1}\right]} & \cdots & s_{\phi\left[p_{k}, q_{k^{\prime}-1}\right]} & 0 & 0 & \cdots & 0 \\
s_{\phi\left[p_{k+1}, i\right] \uparrow \phi\left[i+1, q_{1}\right]} & \cdots & s_{\phi\left[p_{k+1}, i\right] \uparrow \phi\left[i+1, q_{k^{\prime}-1}\right]} & s_{\phi\left[p_{k+1}, i\right]} & s_{\phi\left[p_{k+1}, q_{k^{\prime}+1}\right]} & \cdots & s_{\phi\left[p_{k+1}, q_{m}\right]} \\
\vdots & \vdots & \vdots & \vdots & \vdots & \vdots & \vdots \\
s_{\phi\left[p_{m}, i\right] \uparrow \phi\left[i+1, q_{1}\right]} & \cdots & s_{\phi\left[p_{m}, i\right] \uparrow \phi\left[i+1, q_{k^{\prime}-1}\right]} & s_{\phi\left[p_{m}, i\right]} & s_{\phi\left[p_{m}, q_{k^{\prime}+1}\right]} & \cdots & s_{\phi\left[p_{m}, q_{m}\right]}
\end{array}\right),
$$

and the canonical matrix $C\left(\Pi^{\prime}\right)$ has the following form

$$
\left(\begin{array}{ccccccc}
s_{\phi\left[p_{1}, q_{1}\right]} & \cdots & s_{\phi\left[p_{1}, q_{k^{\prime}-1}\right]} & 0 & 0 & \cdots & 0 \\
\vdots & \vdots & \vdots & \vdots & \vdots & \vdots & \vdots \\
s_{\phi\left[p_{k}, q_{1}\right]} & \cdots & s_{\phi\left[p_{k}, q_{k^{\prime}-1}\right]} & 0 & 0 & \cdots & 0 \\
s_{\phi\left[p_{k+1}, i\right] \triangleright \phi\left[i+1, q_{1}\right]} & \cdots & s_{\phi\left[p_{k+1}, i\right] \triangleright \phi\left[i+1, q_{k^{\prime}-1}\right]} & s_{\phi\left[p_{k+1}, i\right]} & s_{\phi\left[p_{k+1}, q_{k^{\prime}+1}\right]} & \cdots & s_{\phi\left[p_{k+1}, q_{m}\right]} \\
\vdots & \vdots & \vdots & \vdots & \vdots & \vdots & \vdots \\
s_{\phi\left[p_{m}, i\right] \triangleright \phi\left[i+1, q_{1}\right]} & \cdots & s_{\phi\left[p_{m}, i\right] \triangleright \phi\left[i+1, q_{k^{\prime}-1}\right]} & s_{\phi\left[p_{m}, i\right]} & s_{\phi\left[p_{m}, q_{k^{\prime}+1}\right]} & \cdots & s_{\phi\left[p_{m}, q_{m}\right]}
\end{array}\right) .
$$


For $j: 1 \leq j \leq k^{\prime}-1$ subtracting the $k^{\prime}$-th column of $C\left(\Pi^{\prime}\right)$ multiplied by $s_{\phi\left[i+1, q_{j}\right]}$ from the $j$-th column, and then multiplying -1 for the last $m-k$ rows and the last $m-k^{\prime}+1$ columns, we obtain the matrix $C(\Pi)$. This implies that $C(\Pi)$ and $C\left(\Pi^{\prime}\right)$ are stably equivalent over $\Lambda$.

(d) $i \notin Q_{\Pi}, i+1 \in P_{\Pi}, P_{\Pi^{\prime}}=P_{\Pi}$ and $Q_{\Pi^{\prime}}=Q_{\Pi}$. We omit the proof here since it is similar to Case (c).

\section{Jacobi-Trudi matrices}

In this section we will prove that the Jabobi-Trudi matrix and the transpose of the dual Jacobi-Trudi matrix, for a general skew partition $\lambda / \mu$, are stably equivalent over the ring $\Lambda$ of symmetric functions. Theorem 3.4 states that this is true when $\lambda / \mu$ is edgewise connected, where we do not allow the existence of empty strips in the outside decomposition $\Pi$. The Jacobi-Trudi matrix $J_{\lambda / \mu}$ corresponds to the Giambelli-type matrix $M(\Pi)$ when the cutting strip $\phi$ of $\Pi$ is a horizontal border strip, and the dual Jacobi-Trudi matrix $D_{\lambda / \mu}$ corresponds to the transpose of the matrix $M(\Pi)$ when $\phi$ is a vertical border strip.

For a general skew partition $\lambda / \mu$, we need to be more careful when dealing with the empty strip. Let $c_{\min }=-\lambda_{1}^{\prime}+1$ and $c_{\max }=\lambda_{1}-1$. Let $\phi_{h}$ (or $\phi_{e}$ ) be the horizontal (resp. vertical) border strip starting with the box having content $c_{\min }$ and ending with the box having content $c_{\max }$. Let $\Pi_{h}=\left(\theta_{1}, \cdots, \theta_{\ell(\lambda)}\right)$ be the horizontal outside decomposition of $\lambda / \mu$, where $\theta_{i}$ is a horizontal strip of row $i$ from the $\left(\mu_{i}+1\right)$-st box to the $\lambda_{i}$-th box. When $\lambda_{i}=\mu_{i}$, we take $\theta_{i}$ to be the empty strip. Clearly, each $\theta_{i}$ corresponds to a substrip $\phi_{h}\left[\mu_{i}-i+1, \lambda_{i}-i\right]$ of $\phi_{h}$. Now we see that the Jacobi-Trudi matrix $J_{\lambda / \mu}$ coincides with the transpose of the Giambelli-type matrix $M\left(\Pi_{h}\right)$ defined in (3.5). Similarly, let $\Pi_{e}=\left(\theta_{1}^{\prime}, \cdots, \theta_{\lambda_{1}}^{\prime}\right)$ be the vertical outside decomposition of $\lambda / \mu$, where $\theta_{i}^{\prime}$ is a vertical strip of column $i$ from the $\lambda_{i}^{\prime}$-th box to the $\left(\mu_{i}^{\prime}+1\right)$-st box. When $\lambda_{i}^{\prime}=\mu_{i}^{\prime}$, we take $\theta_{i}^{\prime}$ as the empty strip. Clearly, each $\theta_{i}^{\prime}$ corresponds to a substrip $\phi_{e}\left[-\lambda_{i}^{\prime}+i,-\mu_{i}^{\prime}+i-1\right]$ of $\phi_{e}$. Then the dual Jacobi-Trudi matrix $D_{\lambda / \mu}$ coincides, under permutation of rows and columns, with the Giambelli-type matrix $M\left(\Pi_{e}\right)$. See the first and last matrices of the appendix for an example. The following lemma is straightforward.

Lemma 4.1 Let $\lambda / \mu$ be a partition with $\lambda_{1}^{\prime}=\mu_{1}^{\prime}$. Let $\rho / \nu$ be the skew partition obtained by removing the first column of the skew diagram $\lambda / \mu$. Then the Jacobi-Trudi matrices of $\lambda / \mu$ and $\rho / \nu$ are stably equivalent over $\Lambda$, and so are the dual Jacobi-Trudi matrices.

Therefore, we may assume that $\lambda_{1}^{\prime} \neq \mu_{1}^{\prime}$. Let $\Pi$ be an outside decomposition of $\lambda / \mu$, and let $\phi$ be the cutting strip of $\Pi$. For $i: c_{\min } \leq i \leq c_{\max }$, let $\omega_{i}$ denote the twist transformation at the box of content $i$ from the right direction to the up direction. Now we define the outside decomposition $\omega_{i}(\Pi)$ by the following rule: 
(a') If $\lambda / \mu$ has both a box with content $i$ and a box with content $i+1$, then let $\omega_{i}(\Pi)=$ $\left(\Pi \backslash \Pi^{(i)}\right) \cup \omega_{i}\left(\Pi^{(i)}\right)$, where $\Pi^{(i)}$ is the outside decomposition of the edgewise connected region of $\lambda / \mu$ which has a box with content $i$ and $\omega_{i}\left(\Pi^{(i)}\right)$ is defined as in Section 2.

(b') If $\lambda / \mu$ has a box with content $i$ and but it does not have a box with content $i+1$, then let $\omega_{i}(\Pi)=\Pi$.

(c') If $\lambda / \mu$ has neither a box with content $i$ nor a box with content $i+1$ while it has a box with content less than $i$, then let $\omega_{i}(\Pi)=\Pi \cup\{\phi[i+1, i]\}$ if $\phi[i+1, i] \notin \Pi$, otherwise let $\omega_{i}(\Pi)=\Pi \backslash\{\phi[i+1, i]\}$.

(d') If $\lambda / \mu$ has a box with content $i+1$ and a box with content less than $i$, but it does not have a box with content $i$, then let $\omega_{i}(\Pi)=\Pi$.

The following lemma is a direct verification of the action of $\omega_{i}$ on outside decompositions

Lemma 4.2 Let $\lambda / \mu$ be a skew partition with $\lambda_{1}^{\prime} \neq \mu_{1}^{\prime}$. Let $\Pi_{h}$ and $\Pi_{e}$ be the horizontal outside decomposition and the vertical outside decomposition of $\lambda / \mu$ respectively. Then

$$
\Pi_{e}=\omega_{c_{\max }-1}\left(\omega_{c_{\max }-2}\left(\cdots\left(\omega_{c_{\min }}\left(\Pi_{h}\right)\right) \cdots\right)\right) .
$$

We now reach the following conclusion as an answer to Kuperberg's problem [3, Question 15].

Theorem 4.3 For a skew partition $\lambda / \mu$, the Jacobi-Trudi matrix $J_{\lambda / \mu}$ and $D_{\lambda / \mu}^{T}$ are stably equivalent over the ring of symmetric functions.

Proof. Clearly, $J_{\lambda / \mu}$ and $D_{\lambda / \mu}^{T}$ are stably equivalent if and only if $J_{\lambda / \mu}^{T}$ and $D_{\lambda / \mu}$ are stably equivalent. Due to Lemma 3.2, we only need to prove that the canonical matrices $C\left(\Pi_{h}\right)$ and $C\left(\Pi_{e}\right)$ are stably equivalent over $\Lambda$. Due to Lemma 4.1 , we only deal with the case of $\lambda_{1}^{\prime} \neq \mu_{1}^{\prime}$. By Lemma 4.2 , it suffices to prove that $C(\Pi)$ and $C\left(\omega_{i}(\Pi)\right)$ are stably equivalent under any twist transformation $\omega_{i}$ of the above four cases.

Let $P(\Pi)=\left\{p_{1}, p_{2}, \ldots, p_{m}\right\}$ and $Q(\Pi)=\left\{q_{1}, q_{2}, \ldots, q_{m}\right\}$ be strictly decreasing. Now we see the transformations between the matrices according to the type of $\omega_{i}$.

If $\omega_{i}$ is of type (c'), then the proof is similar to the proof of case (a) and (b) in Theorem 3.4 .

For the case of $\omega_{i}$ being of type (a'), the stably equivalent transformation will be one of the cases of the proof of Theorem 3.4.

If $\omega_{i}$ is of type (b'), then $i \in Q(\Pi)$. Now the proof is similar to the proof of case (c) in Theorem 3.4.

If $\omega_{i}$ is of type (d'), then $i+1 \in P(\Pi)$. Now the proof is similar to that of case (d) in Theorem 3.4. 
The above proof only gives the stably equivalent transformations from the JacobiTrudi matrix to the dual Jacobi-Trudi matrix. In fact, we can also transform the dual Jacobi-Trudi matrix into the Jacobi-Trudi matrix since each transformation $\omega_{i}$ can be reversed.

Combining all the above cases, we have completed the proof.

To illustrate the proof of the above theorem, we take $\lambda / \mu=(6,5,3,1) /(4,4,3)$, see the appendix.

Acknowledgments. We are grateful to the referee and Greg Kuperberg for invaluable comments. This work was supported by the 973 Project on Mathematical Mechanization, the Ministry of Education, the Ministry of Science and Technology, and the National Science Foundation of China.

\section{References}

[1] W. Y. C. Chen, G. G. Yan, A. L. B. Yang, Transformations of border strips and schur function determinants, J. Algebraic Combin. 21 (2005), 379-394.

[2] A. M. Hamel, I.P. Goulden, Planar decompositions of tableaux and Schur function determinants, Europ. J. Combin. 16 (1995), 461-477.

[3] G. Kuperberg, Kasteleyn cokernels, Electron. J. Combin. 9 (2002), \#R29.

[4] M. P. Schützenberger, La correspondance de Robinson, in Combinatoire et représentation du groupe symétrique (Table Ronde, Strasbourg 1976, D. Foata, ed.), Lecture Notes in Math., Vol. 579, Springer, 1977, pp. 59-113.

[5] R. P. Stanley, Enumerative Combinatorics, Vol. II, Cambridge University Press, New York/Cambridge, 1999.

[6] A. Zelevinsky, A generalization of the Littlewood-Richardson rule, J. Algebra 69 (1981), 82-94. 


\section{Appendix}

For the partition $\lambda / \mu=(6,5,3,1) /(4,4,3)$, we have the following Young diagram:

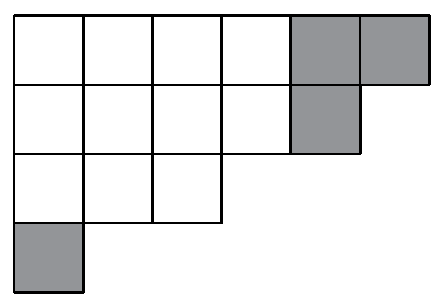

Note that the Jacobi-Trudi matrix $J_{\lambda / \mu}$ is the transpose of the first Giambelli-type matrix, and the dual Jacobi-Trudi matrix $D_{\lambda / \mu}$ is equal to the last Giambelli-type matrix rotated by 180 degrees. Therefore, the dual Jacobi-Trudi matrix can be obtained from the last Giambelli-type matrix by permuting rows and columns. Here we use $[p, q]$ to denote the corresponding border strip in the outside decomposition. The dots in the matrix represent 0 .

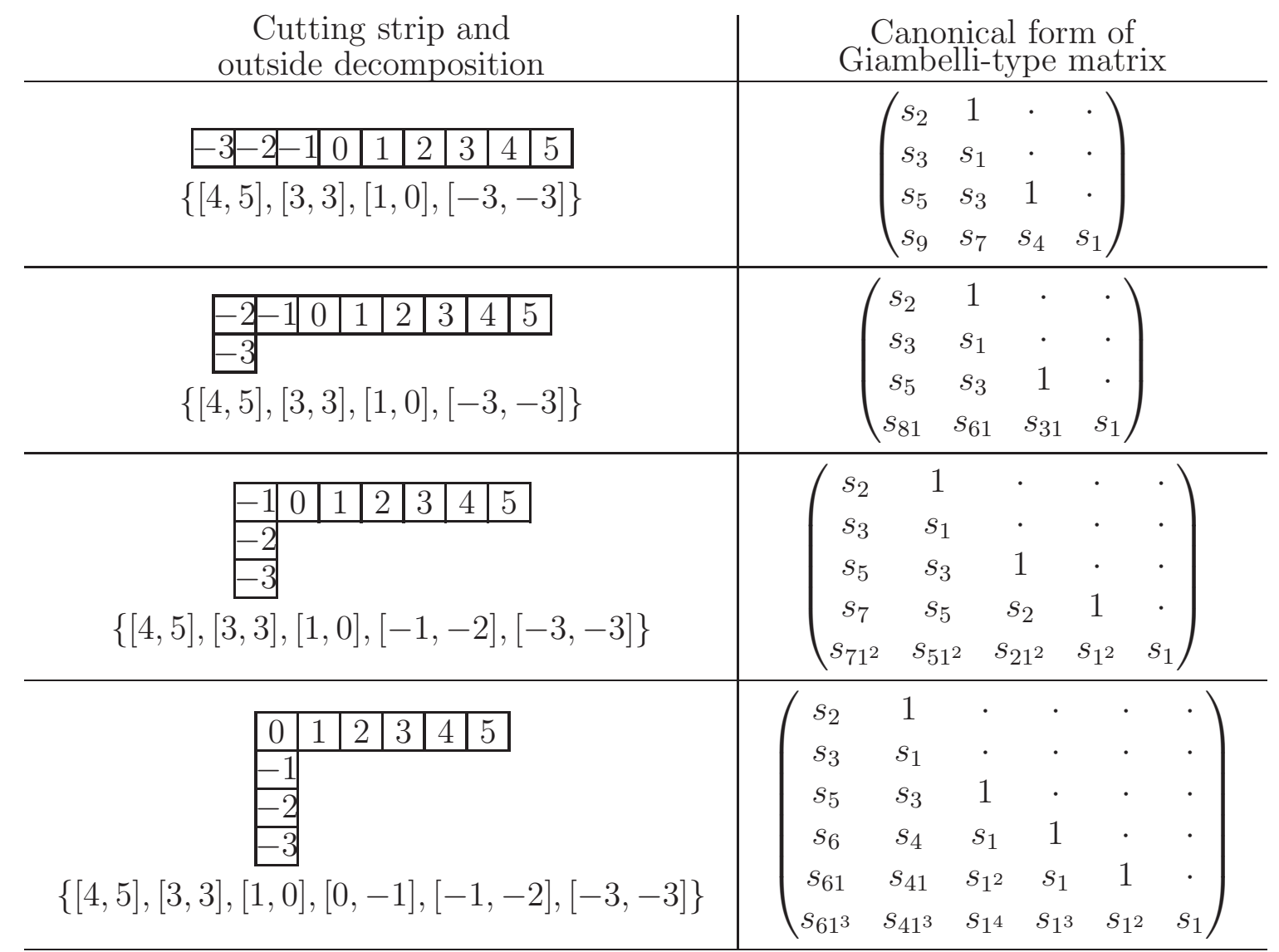

Continuing to the twist transformation, we have the following 


\begin{tabular}{|c|c|}
\hline $\begin{array}{c}\text { Cutting strip and } \\
\text { outside decomposition }\end{array}$ & $\begin{array}{c}\text { Canonical form of } \\
\text { Giambelli-type matrix }\end{array}$ \\
\hline 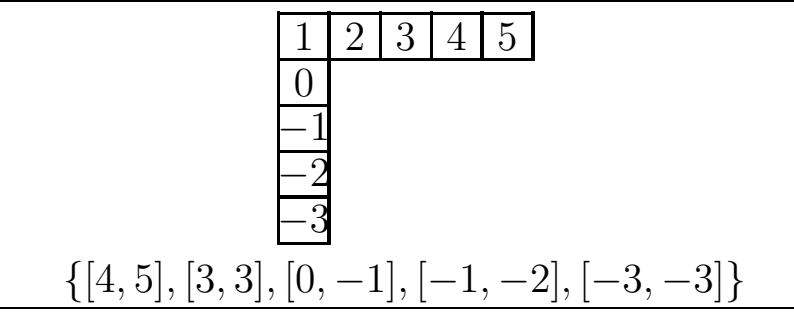 & $\left(\begin{array}{ccccc}s_{2} & 1 & \cdot & \cdot & \cdot \\
s_{3} & s_{1} & \cdot & \cdot & \cdot \\
s_{51} & s_{31} & 1 & \cdot & \cdot \\
s_{51^{2}} & s_{31^{2}} & s_{1} & 1 & \cdot \\
s_{51^{4}} & s_{31^{4}} & s_{1^{3}} & s_{1^{2}} & s_{1}\end{array}\right)$ \\
\hline \begin{tabular}{rl|l|l|l|}
2 & 3 & 4 & 5 \\
1 & & \\
\cline { 1 - 1 } 0 & & \\
\cline { 1 - 1 }-1 & & \\
\cline { 1 - 1 }-2 & \\
-3 & \\
\end{tabular} & $\left(\begin{array}{cccccc}s_{2} & 1 & \cdot & \cdot & \cdot & \cdot \\
s_{3} & s_{1} & \cdot & \cdot & \cdot & \cdot \\
s_{4} & s_{2} & 1 & \cdot & \cdot & \cdot \\
s_{41^{2}} & s_{21^{2}} & s_{1^{2}} & 1 & \cdot & \cdot \\
s_{41^{3}} & s_{21^{3}} & s_{1^{3}} & s_{1} & 1 & \cdot \\
s_{41^{5}} & s_{21^{5}} & s_{1^{5}} & s_{1^{3}} & s_{1^{2}} & s_{1}\end{array}\right)$ \\
\hline 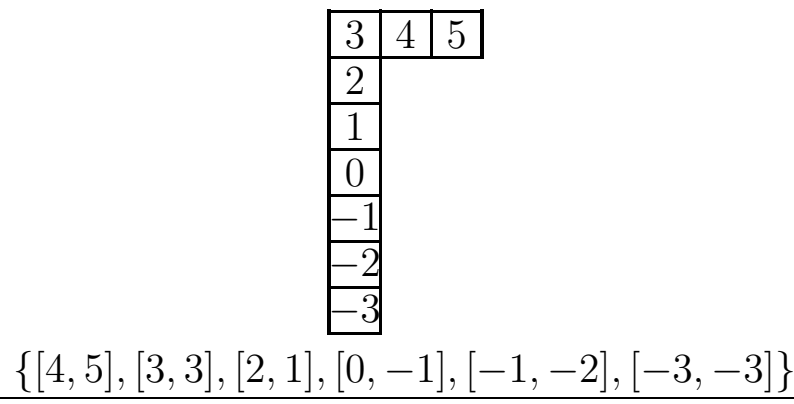 & $\left(\begin{array}{cccccc}s_{2} & 1 & \cdot & \cdot & \cdot & \cdot \\
s_{3} & s_{1} & \cdot & \cdot & \cdot & \cdot \\
s_{31} & s_{1^{2}} & 1 & \cdot & \cdot & \cdot \\
s_{31^{3}} & s_{1^{4}} & s_{1^{2}} & 1 & \cdot & \cdot \\
s_{31^{4}} & s_{1^{5}} & s_{1^{3}} & s_{1} & 1 & \cdot \\
s_{31^{6}} & s_{1^{7}} & s_{1^{5}} & s_{1^{3}} & s_{1^{2}} & s_{1}\end{array}\right)$ \\
\hline 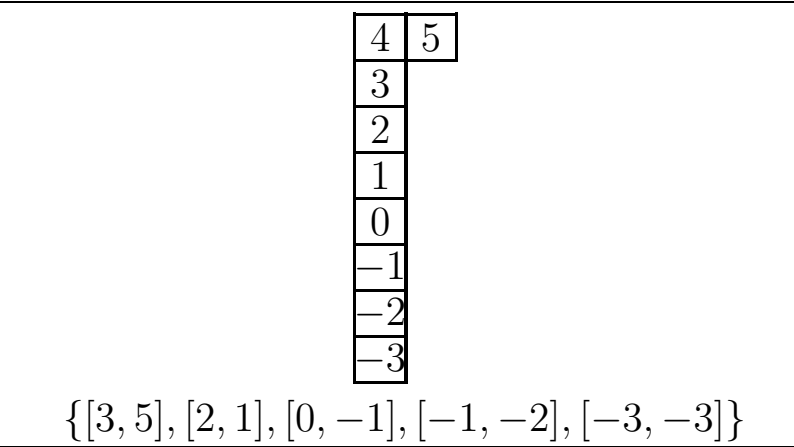 & $\left(\begin{array}{ccccc}s_{21} & \cdot & \cdot & \cdot & \cdot \\
s_{21^{2}} & 1 & \cdot & \cdot & \cdot \\
s_{21^{4}} & s_{1^{2}} & 1 & \cdot & \cdot \\
s_{21^{5}} & s_{1^{3}} & s_{1} & 1 & \cdot \\
s_{21^{7}} & s_{1^{5}} & s_{1^{3}} & s_{1^{2}} & s_{1}\end{array}\right)$ \\
\hline \begin{tabular}{|c|}
5 \\
4 \\
3 \\
2 \\
1 \\
0 \\
-1 \\
-2 \\
-3 \\
$[5,5],[3,4],[2,1],[0,-1],[-1,-2],[-3,-3]\}$ \\
\end{tabular} & $\left(\begin{array}{cccccc}s_{1} & 1 & \cdot & \cdot & \cdot & \cdot \\
s_{1^{3}} & s_{1^{2}} & \cdot & \cdot & \cdot & \cdot \\
s_{1^{4}} & s_{1^{3}} & 1 & \cdot & \cdot & \cdot \\
s_{1^{6}} & s_{1^{5}} & s_{1^{2}} & 1 & \cdot & \cdot \\
s_{1^{7}} & s_{1^{6}} & s_{1^{3}} & s_{1} & 1 & \cdot \\
s_{1^{9}} & s_{1^{8}} & s_{1^{5}} & s_{1^{3}} & s_{1^{2}} & s_{1}\end{array}\right)$ \\
\hline
\end{tabular}

